\section{Diferenças no processo de atenção ao pré-natal entre unidades da Estratégia Saúde da Família e unidades tradicionais em um município da Região Sul do Brasil}

\author{
Differences in prenatal care between health \\ services under the Family Health Strategy and \\ traditional primary care clinics in Rio Grande, \\ Rio Grande do Sul State, Brazil
}

\author{
1 Faculdade de Medicina, \\ Universidade Federal do Rio \\ Grande, Rio Grande, Brasil. \\ 2 Programa de Pós-graduação \\ em Ciências da Saúde, \\ Universidade Federal do Rio \\ Grande, Rio Grande, Brasil. \\ 3 Programa de Pós-graduação \\ em Epidemiologia, \\ Universidade Federal de \\ Pelotas, Pelotas, Brasil. \\ 4 Secretaria Municipal de \\ Saúde de Rio Grande, Rio \\ Grande, Brasil. \\ Correspondência \\ R. A. Mendoza-Sassi \\ Divisão de População e Saúde, \\ Faculdade de Medicina, \\ Universidade Federal do Rio \\ Grande. \\ Rua General Osório s/n, Rio \\ Grande, RS 96200-400, Brasil. \\ rmend@vetorial.net
}

\begin{abstract}
This study aimed to evaluate differences in prenatal care between services under the Family Health Strategy (FHS) and traditional public primary care clinics in Rio Grande, Rio Grande do Sul State, Brazil. A cross-sectional study was performed with all women who gave birth from January 1st to December 31st, 2007, and who received prenatal care in the municipal health system. The procedures recommended by the Ministry of Health were compared according to model of care. Among the 961 pregnant women, those treated under the FHS received a higher percentage of some forms of care (use of ferrous sulfate, tetanus vaccination, and HIV and syphilis tests). Other procedures were also more frequent under the FHS, but failed to reach the recommended levels (breast examination and Pap smear). Measurement of blood pressure, uterine height, and weight were quite frequent in both groups. Identification of pregnant women in the first trimester failed to reach 70\%. Women under the FHS received better care, but some procedures still fell short of expected levels, and efforts are thus needed to improve the quality of prenatal care.
\end{abstract}

Prenatal Care; Delivery Health Care; Evaluation
Raul A. Mendoza-Sassi 1,2

Juraci Almeida Cesar 1,2,3

Tarso Pereira Teixeira 1,4

César Ravache 1

Gerson Donizete Araújo 1

Tatiana Corrêa da Silva 1

\section{Introdução}

Os cuidados no pré-natal constituem uma importante ação programática, uma vez que permitem acompanhar a gravidez da gestante e identificar situações de risco para a mãe ou para o feto, corrigindo-as quando necessário. Uma atenção adequada pode evitar importantes desfechos negativos no recém-nascido, como retardo no crescimento intrauterino, baixo peso ao nascer e prematuridade, contribuindo para a diminuição da mortalidade infantil 1,2,3.

A cobertura à atenção pré-natal tem aumentado no Brasil, e segundo dados do Ministério da Saúde o atendimento pré-natal realizado na atenção básica aumentou em mais de $350 \%$ nos últimos 10 anos (Atendimento pré-natal segundo ano. Sistema de Informação de Atenção Básica. http://tabnet.datasus.gov.br, acessado em 27/ Mar/2010). Essa tendência tem sido corroborada por estudos de coorte no Município de Pelotas, sul do Brasil, que mostram a melhora dos indicadores de cobertura de pré-natal, média de consultas e trimestre de início do pré-natal 4.

Mas apesar dessa tendência de melhora da cobertura e de certos indicadores, as avaliações da qualidade do pré-natal realizadas não têm sido tão alentadoras. Os índices de adequação do pré-natal são baixos, como mostram alguns estudos realizados nos últimos anos 5,6,7,8, e o trimestre de início do acompanhamento ainda apresenta valor inferior ao desejado 5,6,8,9,10. 
A qualidade do pré-natal também tem sido avaliada utilizando-se o conceito de qualidade da atenção de Donabedian 11, considerando a tríade estrutura, processo e resultado, com maior ênfase no processo. Faz parte do processo de atenção o conteúdo dos cuidados realizados durante o pré-natal, e a avaliação é realizada mediante a comparação das práticas observadas com as recomendações do Ministério da Saúde sobre a atenção ao pré-natal, divulgadas por meio de manual técnico 12.

Na opinião de Donabedian ${ }^{13}$, é o cumprimento dessas normas e regras que podem melhorar a qualidade na atenção, quando o foco da análise é o processo, uma vez que orientam os responsáveis pela assistência sobre o cumprimento de determinadas práticas, que, se cumpridas, deverão garantir o melhor resultado possível. Em suma, segundo essa abordagem, a padronização de certos procedimentos e cuidados à saúde da gestante, mediante a utilização de protocolos, vai contribuir com uma atenção de maior qualidade para todos os pacientes.

Os estudos que avaliam o processo do prénatal mostram que nem todas as práticas recomendadas são cumpridas, e algumas, tais como o exame das mamas, o exame ginecológico e a prevenção do câncer de colo encontram-se muito abaixo do desejado 5,6,14,15,16,17.

Parte dos avanços ocorridos na atenção à saúde no Brasil e em particular na atenção pré-natal deve-se à Estratégia Saúde da Família (ESF), que vem substituindo gradativamente o modelo tradicional de atenção primária nas unidades básicas de saúde (UBS). Entre os aspectos que diferenciam a ESF de outros modelos de atenção está o fato de que, a programação de serviços de saúde oferecidos pela ESF à comunidade e a grupos específicos obedece a recomendações e orientações do Ministério da Saúde 18,19. As avaliações realizadas sobre o desempenho da ESF em diversos desfechos mostram que, mediante ações programáticas bem definidas, a estratégia está aumentando a equidade dos cuidados à saúde da população brasileira 9,20,21,22.

No que concerne aos cuidados que a gestante deve receber durante o pré-natal e no parto, as equipes de saúde da família trabalham com normas e regras sobre como deve ser realizada a atenção ao pré-natal, mediante a utilização de protocolo, que contempla todos os procedimentos e exames que devem ser realizados 12 . Já no modelo tradicional de atenção, a programação de serviços de saúde e a atenção à saúde não necessariamente seguem as recomendações do Ministério da Saúde ou utilizam protocolo de atenção. É de se esperar que a atuação das equi- pes de saúde de família, utilizando esse marco normativo, deveria desenvolver um pré-natal de maior qualidade.

A avaliação do desempenho da ESF na atenção pré-natal, comparado ao modelo tradicional, tem sido realizada por poucos estudos $16,23,24$. Os resultados indicam que a estratégia desenvolve melhor os cuidados, mas são necessários novos estudos para avaliar essa situação de forma mais ampla e completa.

A análise do pré-natal no Município de Rio Grande, Rio Grande do Sul, Brasil, brinda uma excelente oportunidade para realizar essa análise do processo, uma vez que apresenta os dois modelos de atenção. A ESF está funcionando desde 2001 com 27 equipes consolidadas, e participa da Avaliação para a Melhoria da Qualidade da Estratégia Saúde da Família (AMQ - http://dtr2002. saude.gov.br/proesf/autoavaliacaoesf/paginas/ amqoquee.asp, acessado em 10/Fev/2011), metodologia implementada pelo Ministério da Saúde para a autoavaliação da estratégia nos municípios. O estudo pode contribuir para o desenvolvimento e aperfeiçoamento da ESF, e acrescentar informações úteis para o planejamento de ações programáticas, dirigidas ao cuidado da gestante e da criança.

\section{Material e métodos}

O presente estudo faz parte de um mais amplo que tinha como objetivo avaliar todos os cuidados perinatais no Município de Rio Grande. Foi um delineamento transversal, realizado entre 1o de janeiro e 31 de dezembro de 2007. Ao longo desse período, todas as gestantes que tiveram seu parto em um dos dois hospitais do município e que deram à luz recém-nascidos com peso superior a $500 \mathrm{~g}$ e com idade gestacional igual ou maior que 20 semanas, foram entrevistadas. A entrevista às gestantes era realizada nas primeiras 24 horas após o parto, mediante um questionário pré-codificado e pré-testado, aplicado por 10 entrevistadores treinados para esta tarefa. O questionário incluiu, entre outras, questões relativas aos aspectos socioeconômicos e demográficos da mãe, procedimentos e exames recomendados pelo Ministério da Saúde e realizados pela gestante, e dados da história clínica do recém-nascido. Dez por cento das gestantes foram entrevistadas novamente, logo após a alta, com a finalidade de avaliar a confiabilidade do instrumento. Os dados foram duplamente digitados no programa Epi Info 6.04d (Centers for Disease Control and Prevention, Atlanta, Estados Unidos), para identificação de possíveis erros de digitação. Posteriormente, o banco de dados 
final passou por uma nova correção para identificar erros de consistência e/ou de amplitude nos valores. Após, foi traduzido para o programa Stata 9.2 (Stata Corp., College Station, Estados Unidos), no qual foram realizadas todas as análises estatísticas.

Do total das 2.591 gestantes, 2.557 cumpriram as condições antes mencionadas e foram incluídas na pesquisa. Para fins do presente estudo foi extraída uma subamostra com todas as mulheres que realizaram o pré-natal na rede pública municipal.

O fator de interesse principal foi o tipo de modelo de atenção no qual a gestante realizou o pré-natal. Essa variável foi criada usando-se a classificação das UBS, onde a gestante referiu realizar o pré-natal, em dois grupos: ESF ou UBS tradicional.

O Município de Rio Grande está localizado no extremo sul do Brasil. A ESF iniciou no ano de 2001 e em 2007 contava com 27 equipes de saúde da família. Em todas, realizava - se o prénatal de baixo risco, dentro da lógica territorial e centrada no cuidado da pessoa, sua família e comunidade. Na prática diária da atenção à saúde as equipes se apoiavam nas recomendações do Ministério da Saúde. Periodicamente, participavam de reuniões nas quais se discutiam temas relacionados aos cuidados em saúde da população, com o intuito de aprimorar estes cuidados. A cobertura de ESF do município, no momento do estudo, estava em 35\% da população (dados da Secretaria Municipal de Saúde de Rio Grande). O restante da população era atendido pela rede pública nas UBS, de atenção básica tradicional, e pelos serviços privados, a partir de convênios e seguros de saúde.

Os indicadores do processo da atenção, sobre os quais se estudou o efeito do modelo de atenção, são os recomendados pelo Ministério da Saúde 12: (1) histórico do pré-natal da mãe incluindo trimestre de início do pré-natal, número de consultas realizadas, internações ocorridas durante a gravidez; (2) exames realizados durante o pré-natal; (3) procedimentos recebidos durante as consultas: medida do peso, da altura uterina, da pressão arterial, exame das mamas, exame ginecológico e prevenção do câncer de colo (apenas nas gestantes que não tinham realizado o exame antes de engravidar); e (4) orientações e recomendações recebidas durante as consultas como amamentação, uso de sulfato ferroso e vacinação contra o tétano (apenas para as gestantes não vacinadas previamente). Foram também estudadas algumas características do recém-nascido, como baixo peso ao nascer (menos de $2.500 \mathrm{~g}$ ), ocorrência de óbito e de internação na UTI neonatal.
A análise consistiu, em um primeiro momento, na comparação das características demográficas e socioeconômicas, de acordo com o modelo de atenção em que se realizou o pré-natal. A seguir, estimou-se a prevalência para cada um dos procedimentos e exames. Nessa fase, a verificação estatística das diferenças foi realizada utilizando-se no caso das variáveis categóricas o teste de qui-quadrado e, no caso das variáveis numéricas o teste de Student ou de Kruskal Wallis.

Como houve diferença sociodemográficas no que se refere à escolaridade, cor da pele e presença de companheiro, decidiu-se realizar uma análise multivariada, ajustando o tipo de modelo de atenção para as características sociodemográficas. No caso do número de consultas e de exames, ajustou-se também para o trimestre de início do pré-natal. Utilizou-se a regressão de Poisson, calculando-se as razões de prevalência (RP) com seus respectivos intervalos de 95\% de confiança (IC95\%) para cada um dos desfechos. Em todas as análises estatísticas optou-se por utilizar um valor de $\mathrm{p}$ inferior a 0,05 para um teste bicaudal. Por estarem sendo testadas várias hipóteses, os valores de $\mathrm{p}$ inferiores a 0,05 foram corrigidos pelo método de Bonferroni.

O projeto de pesquisa foi aprovado pelo comitê de ética em pesquisa na área da Saúde da Universidade Federal do Rio Grande.

\section{Resultados}

Das 2.557 parturientes entrevistadas durante o ano de 2007, 961 realizaram o pré-natal na rede pública municipal $(37,6 \%)$ e constituíram a amostra deste estudo. Dessas, 60,8\% foram atendidas na ESF, enquanto as demais, no modelo tradicional.

As características sociodemográficas da amostra estudada podem ser vistas na Tabela 1 . Os grupos mostraram-se similares em termos de média de idade e na distribuição dos quartis de renda, mas houve diferenças significativas na cor da pele, na presença de companheiro e na escolaridade.

A Tabela 2 mostra as prevalências de procedimentos e exames realizados entre os dois grupos durante o pré-natal, bem como as razão de prevalência (RP) e IC95\% para estes desfechos, entre gestantes atendidas na ESF versus aquelas atendidas no modelo tradicional. Essas análises foram ajustadas para idade, cor, escolaridade, renda, presença de companheiro e trimestre de início do pré-natal.

A prevalência de cesárea foi similar entre os dois grupos e não encontramos diferença estatisticamente significativa. Houve sim uma 
Características sociodemográficas, segundo tipo de atendimento. Rio Grande, Rio Grande do Sul, Brasil, 2007 (N = 961 ).

\begin{tabular}{|c|c|c|c|}
\hline Características & $\operatorname{PSF}(n=584)$ & Tradicional $(n=377)$ & Valor de $p$ \\
\hline Média idade (DP) & $24,3(6,7)$ & $23,9(6,3)$ & 0,3 * \\
\hline Média escolaridade (DP) & $6,6(2,8)$ & $7,4(2,8)$ & $0,0001 *$ \\
\hline \multicolumn{4}{|l|}{ Cor da pele } \\
\hline Branca & $66,8 \%$ & $59,7 \%$ & $0,04 * \star$ \\
\hline Negra & $13,5 \%$ & $18,8 \%$ & \\
\hline Parda/Morena & $19,7 \%$ & $21,5 \%$ & \\
\hline \multicolumn{4}{|c|}{ Quartis de renda com média e DP } \\
\hline 1 으 $(284,01 ; 154,17)$ & $42,0 \%$ & $39,3 \%$ & 0,3 ** \\
\hline 2 으 $(641,78 ; 111,06)$ & $33,9 \%$ & $34,8 \%$ & \\
\hline 3으 $(1047,41 ; 136,89)$ & $17,6 \%$ & $16,2 \%$ & \\
\hline 4으 $(2544,45 ; 1709,80)$ & $6,5 \%$ & $9,8 \%$ & \\
\hline Vive com companheiro & $82,9 \%$ & $76,7 \%$ & 0,02 ** \\
\hline Grávidas adolescentes & $14,04 \%$ & $15,38 \%$ & 0,6 ** \\
\hline
\end{tabular}

DP: desvio-padrão; PSF: Programa Saúde da Família.

* Teste de Student

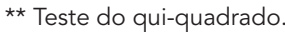

maior proporção de parturientes que foram internadas durante a gestação dentro da ESF (18\% vs. $9 \%$ ), e o risco de se internar após ajuste foi maior que $20 \%$.

Com relação às consultas de pré-natal, $78 \%$ das mulheres atendidas na ESF realizaram seis ou mais consultas, contra $60 \%$ no modelo tradicional ( $\mathrm{p}=0,003$ ), com probabilidade de ocorrência $10 \%$ maior no grupo da ESF ( $p=0,003)$. Da mesma forma, a média de consultas de pré-natal foi maior nesse grupo $(7,5 v s .5,9 ; \mathrm{p}=0,003)$ e a $R P$ para realizar consultas foi $25 \%$ maior.

Nos dois grupos não houve diferença na proporção de gestantes que iniciou o pré-natal no primeiro trimestre. Em ambos os grupos, uma porcentagem superior aos $98 \%$ manifestou intenção de amamentar. Tampouco houve diferença na proporção de gestantes que trouxeram a carteirinha de pré-natal para o hospital. Sobre o planejamento da gravidez, as prevalências foram baixas nos dois grupos (44\% vs. 35\%) e sem diferença estatística. Em referência aos exames realizados durante a gestação (hemograma, exame de urina, exame de HIV e exame para sífilis), os dois grupos apresentaram porcentagens próximas a $100 \%$, não havendo diferenças significativas. Quando analisadas as diferenças entre as médias de exames observou-se, em todos os casos, um resultado significativamente maior no grupo da ESF. A média de exames para HIV foi de 2 no grupo da ESF contra 1,7 no modelo tradicional. A média de exames para sífilis no grupo
ESF foi significativamente maior, mas com um valor inferior a $2(1,4 v s .1,2)$. Após controlar para fatores de confusão, as probabilidades ajustadas de realizar esses exames foram maiores entre as gestantes da ESF, variando de $16 \%$ a $28 \%$, segundo o tipo de exame.

Não houve diferença significativa na realização de ultrassonografia obstétrica, avaliação da altura uterina, peso e pressão arterial. Em todos esses procedimentos a prevalência foi próxima a $100 \%$. Também não houve diferença significativa na realização do exame ginecológico, mas, neste caso, as proporções foram baixas em ambos os grupos $(40,8 \%$ vs. $35,8 \%)$.

A prevalência de uso de sulfato ferroso durante a gestação e a realização da vacina antitetânica foi significativamente maior entre as gestantes do grupo da ESF, cerca de $80 \%$, contra $60 \%$ no outro grupo. A probabilidade de realização desses procedimentos foi superior a $40 \%$ no grupo da ESF. Dois outros quesitos mostraram-se mais frequentes nesse grupo e estatisticamente significativos: o exame de mamas e a prevenção do câncer de colo. Mas apesar de as gestantes da ESF terem uma probabilidade maior de realizar esses procedimentos ( $77 \%$ e $62 \%$, respectivamente), a cobertura para eles não alcançou a metade das gestantes da ESF, e no modelo tradicional atingiu em torno de 1/4 delas. A orientação sobre amamentação foi significativamente superior no grupo atendido na ESF ( $72 \%$ vs. 40\%) e, ajustando para os fatores de confusão, a probabilidade 
Prevalência (P) e razões de prevalência (RP) ajustadas de procedimentos e exames realizados pelas gestantes, segundo modelo de atenção. Rio Grande, Rio Grande do Sul, Brasil, 2007 ( $\mathrm{N}=961)$.

\begin{tabular}{|c|c|c|c|c|c|c|}
\hline Procedimento/exame & $\begin{array}{l}\text { PSF (\%) } \\
{[n=584]}\end{array}$ & $\begin{array}{c}\text { Tradicional (\%) } \\
\quad[n=377]\end{array}$ & Valor de $p$ * & $\mathrm{RP}$ ** & IC95\% & $\mathrm{P} \star \star \star \star$ \\
\hline Fez cesárea & 39,6 & 36,1 & 0,3 & 1,11 & $0,89-1,38$ & 0,3 \\
\hline Internou-se durante gestação & 18,8 & 9,0 & $0,003 \#$ & 2,0 & $1,39-2,88$ & $0,003 \#$ \\
\hline Fez 6 ou mais consultas de pré-natal & 77,7 & 60,2 & $0,003 \#$ & $1,10 \# \#$ & $1,07-1,14$ & $0,003 \#$ \\
\hline Média de consultas pré-natal & $7,5(2,9)$ & $5,9(2,2)$ & $0,003 \#$ & $1,25 \# \#$ & $1,20-1,31$ & $0,003 \#$ \\
\hline Início pré-natal 1ㅇ trimestre & 69,4 & 64,2 & 0,09 & 1,12 & $1,01-1,24$ & $0,9 \#$ \\
\hline Pretende amamentar & 98,6 & 98,9 & 0,7 & 1,00 & $0,88-1,14$ & 0,9 \\
\hline Tinha carteira pré-natal no hospital & 71,4 & 68,4 & 0,3 & 1,06 & $0,90-1,24$ & 0,5 \\
\hline Planejou filho & 43,7 & 35,0 & $0,2 \#$ & 1,20 & $1,02-1,41$ & $0,9 \#$ \\
\hline Fez hemograma & 99,5 & 99,2 & 0,6 & 1,00 & $0,99-1,01$ & 0,7 \\
\hline Média hemogramas (DP) & $2,7(1,5)$ & $1,5(1,1)$ & 0,003 \#, \#\#\# & $1,23 \# \#$ & $1,14-1,32$ & $0,003 \#$ \\
\hline Fez exame de urina & 98,6 & 97,4 & 0,4 & 1,00 & $0,99-1,03$ & 0,3 \\
\hline Média de exames urina (DP) & $2,6(1,5)$ & $1,9(1,1)$ & 0,003 \#, \#\#\# & 1,28 \#\# & $1,19-1,38$ & 0,003 \\
\hline Fez exame HIV & 99,5 & 99,2 & 0,6 & 1,00 & $0,99-1,02$ & 0,5 \\
\hline Média de exame HIV & $2,0(0,96)$ & $1,7(0,67)$ & 0,003 \#, \#\#\# & $1,16 \# \#$ & $1,09-1,22$ & $0,003 \#$ \\
\hline Fez exame para sífilis & 99,5 & 99,2 & 0,6 & 1,0 & $0,88-1,14$ & 0,9 \\
\hline Média exame para sífilis & $1,4(0,94)$ & $1,2(0,59)$ & 0,003 \#, \#\# & 1,22 \#\# & $1,13-1,31$ & 0,003 \\
\hline Fez ultrassom & 92,5 & 91,2 & 0,7 & 1,01 & $0,97-1,05$ & 0,5 \\
\hline Média de ultrassom & $1,6(1,1)$ & $1,7(1,0)$ & $0,6 *$, \# & $0,96 \# \#$ & $0,89-1,04$ & 0,4 \\
\hline Medição altura uterina & 99,5 & 99,7 & 0,7 & 1,00 & $0,99-1,01$ & 0,9 \\
\hline Foi pesada & 98,7 & 99,7 & $0,9 \#$ & 0,99 & $0,98-1,00$ & 0,2 \\
\hline Medição de pressão arterial & 99,5 & 97,9 & $0,6 \#$ & 1,02 & $0,99-1,03$ & 0,06 \\
\hline Exame ginecológico & 40,8 & 35,8 & 0,1 & 1,16 & $0,98-1,37$ & 0,08 \\
\hline Usou sulfato ferroso & 82,0 & 56,2 & $0,003 \#$ & 1,47 & $1,33-1,61$ & $0,003 \#$ \\
\hline $\begin{array}{l}\text { Recebeu vacina antitetânica (somente não vacinadas } \\
\text { previamente) }\end{array}$ & 89,6 & 62,7 & $0,003 \#$ & 1,44 & $1,29-1,61$ & $0,003 \#$ \\
\hline Teve as mamas examinadas & 45,7 & 26,0 & $0,003 \#$ & 1,77 & $1,46-2,14$ & 0,003 \\
\hline $\begin{array}{l}\text { Fez prevenção câncer de colo uterino durante } \\
\text { gravidez (excluídas aquelas que fizeram antes de } \\
\text { engravidar) }\end{array}$ & 46,6 & 28,9 & $0,003 \#$ & 1,62 & $1,34-1,97$ & $0,003 \#$ \\
\hline Recebeu orientação sobre amamentação & 71,8 & 39,6 & $0,003 \#$ & 1,81 & $1,58-2,07$ & 0,003 \\
\hline Recebeu orientação sobre exercício físico & 62,3 & 58,5 & 0,2 & 1,07 & $0,96-1,19$ & 0,2 \\
\hline Recém-nascido com baixo peso ao nascer & 8,9 & 9,8 & 0,6 & 0,87 & $0,56-1,33$ & 0,5 \\
\hline Óbito do recém-nascido & 1,5 & 2,1 & 0,5 & 1,00 & $0,88-1,15$ & 0,9 \\
\hline $\begin{array}{l}\text { Internou-se na unidade de tratamento intensivo } \\
\text { neonatal }\end{array}$ & 3,6 & 3,7 & 0,9 & 0,98 & $0,51-1,90$ & 0,9 \\
\hline
\end{tabular}

DP: desvio-padrão; PSF: Programa Saúde da Família.

* Teste do qui-quadrado;

** Ajustados para idade, cor, estado civil, escolaridade e renda;

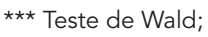

\# Valores corrigidos pelo método de Bonferroni;

\#\# Ajustados para idade, cor, estado civil, escolaridade, renda e trimestre de início do pré-natal;

\#\#\# Teste de Kruskal Wallis. 
de a gestante receber orientação foi $80 \%$ maior ( $p=0,003$ ). Já a orientação sobre a realização de exercícios foi similar nos grupos, situando-se em torno de $60 \%$.

As prevalências de peso ao nascer inferior a $2.500 \mathrm{~g}$, ocorrência de óbito do recém-nascido e internação na UTI neonatal foram similares e não tiveram diferenças significativas.

\section{Discussão}

O presente estudo avaliou o processo de atenção pré-natal na rede pública municipal de Rio Grande, utilizando as normas do Ministério da Saúde. Encontramos um desempenho superior da ESF comparado ao modelo tradicional, em alguns procedimentos, mas também identificamos que existem outros que estão aquém do valor esperado, mostrando que as práticas dos profissionais de saúde na atenção ao pré-natal devem continuar sendo aperfeiçoadas.

Antes de prosseguir é necessário assinalar algumas limitações metodológicas que podem ter afetado os resultados aqui apresentados. $\mathrm{O}$ delineamento transversal utilizado para este tipo de avaliação não apresentou problema quanto ao estabelecimento da relação temporal entre a ocorrência do fator principal e do desfecho (causalidade reversa), uma vez que o fator de interesse era o tipo de modelo de atenção, e o desfecho o processo da atenção. Em relação aos fatores de confusão (sociodemográficos) utilizados no ajuste, é pouco provável que tenham sofrido mudanças importantes ao longo da gestação. Deve-se considerar também a possibilidade de viés de memória. Pode-se pensar que as gestantes atendidas na ESF, em função do vínculo com a equipe, recordem melhor dos exames e dos procedimentos realizados e, neste caso, haveria uma superestimação das prevalências e dos riscos relativos encontrados. Contudo, houve vários procedimentos nos quais a maior parte das gestantes de ambos os grupos recordaram ter realizado. Assim, se houvesse esse tipo de viés, este deveria ser seletivo para apenas algum tipo de desfecho em particular, o que é menos provável de ocorrer

Com relação ao poder estatístico do estudo, como esta pesquisa foi realizada com base em um estudo maior, não foi realizado um cálculo de amostra para estimar as diferenças esperadas. Os cálculos posteriores mostraram que, com o tamanho da amostra estudada, se teria um poder igual ou superior a $80 \%$ para encontrar uma diferença de $10 \%$ ou maior entre duas proporções. Contudo, pode ter ocorrido falta de poder estatístico para algumas associações nas quais as diferenças foram menores que $10 \%$. Mas, como nesses casos, a maior parte das diferenças entre os grupos em que não houve associação significativa foi pequena e com intervalos de confiança estreitos, a falta de poder estatístico parece ser mais remota. Algumas associações inicialmente significativas perderam essa condição ao realizarmos a correção pelo método de Bonferroni.

Na análise da atenção pré-natal foi identificado um conjunto de procedimentos que alcançou valores próximos a $100 \%$ nos dois grupos, e onde se encontram a aferição do peso, a medida da pressão arterial e da altura uterina. Estudos realizados em outros locais e em outros anos também encontraram porcentuais elevados para esses mesmos procedimentos 5,8,10. Essa consistência nos resultados entre os estudos está indicando que esses procedimentos estão integrados na rotina da atenção pré-natal, tanto nos profissionais da ESF quanto nos que trabalham no modelo tradicional.

O mesmo ocorre quando se avalia a realização de exames laboratoriais. Os dois modelos de atenção atingiram mais de $90 \%$ de cobertura para exames como hemograma, exame qualitativo de urina, sorologia para sífilis e HIV. Outros estudos também acharam uma boa cobertura para esses exames 5,8,10,16,25, mas alguns apresentaram porcentagens inferiores na sorologia para HIV e sífilis 10,16. Há novamente consistência nos estudos ao mostrar que os exames de rotina são solicitados para a maioria das gestantes pelos profissionais de saúde do SUS, mas com variações para HIV e sífilis, dependendo do local do estudo.

Nossa pesquisa avaliou também a média de exames. Nesse caso, os resultados foram significativamente maiores no grupo atendido pela ESF, e mantiveram-se após o ajuste com os fatores de confusão idade, cor da pele, escolaridade, renda e presença de companheiro. Quanto ao exame para HIV, as gestantes da ESF realizaram em média dois exames, o que estaria dentro do recomendado pelo Ministério da Saúde. Já a média de sorologia para sífilis, ajustada também para o trimestre de início do pré-natal, situouse abaixo do valor recomendado pelo mesmo Ministério 12.

Houve um segundo grupo de práticas, no qual as gestantes atendidas dentro da ESF receberam mais cuidados que no modelo tradicional, e esta diferença se manteve na análise ajustada. Nesse grupo estão incluídos a cobertura de vacinação antitetânica, o uso do sulfato ferroso e a orientação sobre a amamentação. Nos dois primeiros, as porcentagens observadas na ESF são, inclusive, ligeiramente superiores àquelas encontradas no total das gestantes atendidas no município (89,6 vs. $78,3 \%$ e $82 \%$ 
vs. $61,7 \%$, respectivamente) ${ }^{26}$, mostrando que a ESF pode ter uma qualidade similar ou superior a outros serviços de saúde. A orientação sobre a amamentação tem um significado especial porque predispõe a gestante ao aleitamento exclusivo, obtendo assim benefícios nutricionais e diminuindo a morbi-mortalidade da criança. $\mathrm{O}$ presente estudo encontrou uma prevalência de $72 \%$, valor muito superior ao observado no modelo tradicional $(40 \%)$ e muito próximo ao encontrado em outro estudo que avaliou diferenças entre a ESF e o modelo tradicional em vários municípios do Rio Grande do Sul 27. Esse melhor desempenho da ESF nessas práticas indica que as equipes de Saúde da Família realizam o processo do pré-natal de uma forma mais completa, incluindo práticas educativas, como orientação sobre a amamentação. A recomendação sobre o exercício físico, que faz parte das informações que devem ser repassadas às gestantes durante o pré-natal, teve uma cobertura de $60 \%$ nos dois grupos. Isso pode indicar que na ESF ainda não se dá a devida importância a essa orientação. No modelo tradicional, o resultado pode evidenciar que é mais fácil para os profissionais de saúde que trabalham nele orientar sobre a realização de exercícios físicos do que fazê-lo para amamentar, o que exige um maior conhecimento de técnicas de aleitamento materno.

Há um terceiro grupo de procedimentos no qual se encontram a prevenção do câncer de colo e o exame das mamas, em que a ESF apresentou melhores resultados e suas gestantes indicaram maiores probabilidades de realização. Mas essa chance maior ocorreu com prevalências muito baixas, tanto na ESF, onde metade recebeu os procedimentos quanto no modelo tradicional, em que apenas um quarto das gestantes os fez. Um dos estudos que também avaliou os dois modelos de atenção, realizado em Montes Claros, Minas Gerais 23 , teve o mesmo resultado para a prevenção do câncer do colo, com porcentagens muito similares ao encontrado em nosso estudo. Outra pesquisa, que também avaliou a diferença entre os modelos em vários municípios brasileiros, encontrou baixas coberturas para a prevenção do câncer de colo na ESF, mas não identificou diferenças significativas com o modelo tradicional 16. O baixo desempenho para esses indicadores se repete em estudos que avaliaram o pré-natal do SUS, em outras localidades 5,10,14,15, indicando que essas práticas apresentam dificuldades em ser incorporadas à rotina do pré-natal, independentemente do local, e que devem ser estudadas formas para modificar essa situação.

Em relação à captação da gestante para realização do pré-natal, a proporção delas que iniciam no primeiro trimestre é de $2 / 3$ nos dois modelos. Outros estudos, apesar das diferenças regionais e temporais, encontraram porcentagens similares e eventualmente mais baixas $7,9,14,25,28$. Isso pode sinalizar que os serviços de saúde, em geral, e a ESF em particular, deveriam avaliar melhor as estratégias utilizadas para obter o afluxo das gestantes ao pré-natal, e entender melhor o comportamento em saúde que elas apresentam 29,30, para dar uma resposta mais adequada às suas necessidades.

Houve sim, uma diferença significativa na proporção de gestantes que realizaram seis ou mais consultas e na média de consultas realizadas no grupo da ESF. Esse resultado também foi encontrado pelo estudo antes citado, que comparou os dois modelos em um município de Minas Gerais 23. É possível que essa maior cobertura de pré-natal na Estratégia obedeça ao trabalho integrado da equipe de saúde, incluindo os agentes comunitários e a existência de grupos de gestantes participando de atividades de promoção de saúde 24 .

Nos desfechos estudados do recém-nascido (baixo peso ao nascer e internação) não houve qualquer influência do tipo de modelo. Foi observado que o efeito da adequação do pré-natal sobre alguns desses desfechos não é inequívoco ${ }^{31}$. De certa forma, isso é esperado porque o processo de atenção ao parto não se limita ao pré-natal e também porque há fatores de risco, como os sociodemográficos ou alguns hábitos e costumes das gestantes, sobre os quais os serviços de saúde têm pouca ou nenhuma possibilidade de remoção durante o pré-natal. Também não se observou nenhum efeito da ESF sobre a proporção de cesáreas, e isto obedece ao argumento anterior. A atenção ao parto não é necessariamente realizado pelo médico que fez o pré-natal, enquanto a ocorrência de cesárea sem indicação técnica depende da gestante e do médico assistente 32,33 .

Entre os resultados observados, chamou a atenção o aumento da proporção de internações e do risco de internar, entre as gestantes atendidas pela ESF. Não há motivos para pensar que as gestantes da ESF apresentam uma maior probabilidade de gravidez de risco ou de comorbidades do que as atendidas no modelo tradicional. Do ponto de vista sociodemográfico os dois grupos eram parecidos e foi realizado o ajuste para fatores de confusão. Pode-se sugerir, então, que haveria uma propensão maior de encaminhamento para internação de gestantes por parte das equipes da estratégia, o que, por sua vez, pode ocorrer por um sistema de referência mais aprimorado dentro da ESF. Como não existem mais informações para analisar melhor essa situação não é possível extrair mais conclusões. 


\section{Conclusões}

O presente artigo mostrou a existência de um processo de atenção ao pré-natal mais completo no modelo da ESF quando comparado com o tradicional, igual a outros estudos que compararam os dois modelos em nível nacional ou regional 9,16,23. Também, assim como esses estudos, identificou vários procedimentos que não são realizados na rotina dentro da ESF e que exigem correções.

Analisando o conjunto dos procedimentos que fazem parte do processo de atenção prénatal, e levando em conta o resultado dos outros estudos realizados sobre o tema, identificou-se que há um grupo de exames e procedimentos que estão consolidados nos cuidados do pré-natal e independem do modelo de atenção. Nesse grupo, situam-se os procedimentos de medidas e exames. Há outro grupo de procedimentos que é mais frequentemente realizado entre as gestantes atendidas na ESF. Nele, os procedimentos são do tipo preventivo e educativo, como o uso de sulfato ferroso, vacinação antitetânica e orientações sobre a promoção de práticas saudáveis. Finalmente, existe um terceiro grupo de práticas mais aplicadas nas gestantes da ESF, mas as frequências são baixas em ambos os grupos e deveriam ser foco de atenção das autoridades em saúde. Nesse grupo, encontram-se os procedimentos que por não estarem diretamente relacionados à gestação são negligenciados pelos profissionais de saúde, como a prevenção do câncer de colo e o exame de mamas.
Os resultados obtidos permitem então classificar os procedimentos segundo a maior ou menor adesão à prática pelos profissionais de saúde. Esses achados devem ser corroborados por futuros estudos e analisados com maior profundidade.

Outra contribuição do estudo é que fornece informações importantes que permitem planejar, de forma mais adequada, os programas de educação continuada com os profissionais de saúde. Deve-se dar maior ênfase àqueles procedimentos que apresentam baixa porcentagem de realização. É necessário sensibilizar todos os profissionais de saúde, tanto aqueles que atuam na ESF quanto os que o fazem no modelo tradicional, acerca da importância de seguir o protocolo de atenção pré-natal recomendado e sobre a padronização dos cuidados à gestante, de forma a garantir uma boa qualidade da atenção pré-natal.

Já outros problemas, como a baixa porcentagem de início do pré-natal no primeiro trimestre e o elevado número de cesáreas, por suas características, exigem uma abordagem diferente. No primeiro caso, há grande influência do comportamento em saúde das gestantes e da possibilidade do sistema incluí-la precocemente nos cuidados de pré-natal. No segundo, deve ser mais estudada a relação gestante/profissional de saúde. As estratégias para uma mudança devem ser pesquisadas e analisadas levando-se em conta a complexidade dessas situações.

\section{Resumo}

Avaliar diferenças na atenção ao pré-natal entre a Estratégia Saúde da Família (ESF) e as unidades básicas de saúde (UBS) tradicionais em Rio Grande, Rio Grande do Sul, Brasil. Foi realizado um estudo transversal com todas as mulheres grávidas que tiveram partos entre 1 o de janeiro e 31 de dezembro de 2007 e fizeram o pré-natal na rede municipal. Compararam-se os procedimentos recomendados pelo Ministério da Saúde, segundo modelo de atenção. Dentre as 961 grávidas, as da ESF receberam em maior percentual alguns cuidados (uso de sulfato ferroso, vacina antitetânica, número de exames para HIV e sífilis). Outros procedi- mentos foram mais frequentes na ESF, mas estavam abaixo do valor recomendado (exame das mamas $e$ prevenção do câncer cervical). As medidas de pressão arterial, altura uterina e de peso foram muito frequentes nos dois grupos. A identificação de gestantes no primeiro trimestre não alcançou $70 \%$. As mulheres da ESF têm um processo de atenção melhor, mas alguns dos procedimentos ainda estão abaixo das expectativas, sendo necessários mais esforços para melhorar a qualidade do pré-natal.

Cuidado Pré-Natal; Assistência à Saúde; Avaliação 


\section{Colaboradores}

R. A. Mendoza-Sassi e J. A. Cesar contribuíram com o planejamento da pesquisa, coleta e análise dos dados, redação do manuscrito e revisão da versão final. T. P. Teixeira, C. Ravache, G. D. Araújo e T. C. Silva colaboraram na análise dos dados, redação do manuscrito e revisão da versão final.

\section{Agradecimentos}

Os autores agradecem o apoio recebido do Fundo das Nações Unidas para a Infância, Pastoral da Criança e da Secretaria Municipal de Saúde de Rio Grande. O estudo foi financiado pela Fundação de Amparo à Pesquisa do Estudo do Rio Grande do Sul (FAPERGS/PPSUS; processo 070090)

\section{Referências}

1. Ferraz EM, Gray RH, Cunha TM. Determinants of preterm delivery and intrauterine growth retardation in north-east Brazil. Int J Epidemiol 1990; 19:101-8.

2. Kilsztajn S, Rossbach A, do Carmo MS, Sugahara GT. Assistência pré-natal, baixo peso e prematuridade no Estado de São Paulo, Brasil, 2000. Rev Saúde Pública 2003; 37:303-10.

3. Wehby GL, Murray JC, Castilla EE, Lopez-Camelo JS, Ohsfeldt RL. Prenatal care effectiveness and utilization in Brazil. Health Policy Plan 2009; 24:175-88.

4. Cesar JA, Matijasevich A, Santos IS, Barros AJ, Dias-da-Costa JS, Barros FC, et al. The use of maternal and child health services in three population-based cohorts in Southern Brazil, 1982-2004. Cad Saúde Pública 2008; 24 Suppl 3:S427-36.

5. Silveira DS, Santos IS, Costa JS. Atenção pré-natal na rede básica: uma avaliação da estrutura e do processo. Cad Saúde Pública 2001; 17:131-9.

6. Ribeiro ERO, Guimarães AM, Bettiol H, Lima DD, Almeida ML, Souza L, et al. Risk factors for inadequate prenatal care use in the metropolitan area of Aracaju, Northeast Brazil. BMC Pregnancy Childbirth 2009; 9:31.
7. Coimbra LC, Silva AA, Mochel EG, Alves MT, Ribeiro VS, Aragão VM, et al. Fatores associados à inadequação do uso da assistência pré-natal. Rev Saúde Pública 2003; 37:456-62.

8. Carvalho DS, Novaes HM. Avaliação da implantação de programa de atenção pré-natal no Município de Curitiba, Paraná, Brasil: estudo em coorte de primigestas. Cad Saúde Pública 2004; 20 Suppl 2:S220-30.

9. Facchini L, Piccini R, Tomasei E, Silveira D, Siqueira F, Rodriques M. Desempenho do PSF no Sul e Nordeste do Brasil: avaliação institucional e epidemiológica da Atenção Básica à Saúde. Ciênc Saúde Coletiva 2006; 11:669-81.

10. Coutinho T, Teixeira M, Dain S, Sayd J, Coutinho L. Adequação do processo de assistência pré-natal entre as usuárias do Sistema Único de Saúde em Juiz de Fora-MG. Rev Bras Ginecol Obstet 2003; 25:717-24.

11. Donabedian A. The quality of care. How can it be assessed? JAMA 1988; 260:1743-8. 
12. Departamento de Ações Programáticas Estratégicas, Secretaria de Atenção à Saúde, Ministério da Saúde. Pré-natal e puerpério: atenção qualificada e humanizada - manual técnico. Brasília: Ministério da Saúde; 2005.

13. Donabedian A. La calidad de la atención médica. Definición y métodos de evaluación. In: Donabedian A, editor. Enfoques básicos para la evaluación. Estructura, proceso y resultado. México DF: La Prensa Médica Mexicana; 1984. p. 94-157.

14. Gonçalves CV, Dias-da-Costa JS, Duarte G, Marcolin AC, Garlet G, Sakai AF, et al. Exame clínico das mamas em consultas de pré-natal: análise da cobertura e de fatores associados em município do Rio Grande do Sul, Brasil. Cad Saúde Pública 2008; 24:1783-90.

15. Dias-da-Costa JS, Olinto MT, Bassani D, Marchionatti CR, Bairros FS, Oliveira ML, et al. Desigualdades na realização do exame clínico de mama em São Leopoldo, Rio Grande do Sul, Brasil. Cad Saúde Pública 2007; 23:1603-12.

16. Ribeiro JM, Costa NR, Pinto LFS, Silva PLB. Atenção ao pré-natal na percepção das usuárias do Sistema Único de Saúde: um estudo comparativo. Cad Saúde Pública 2004; 20:534-45.

17. Dias-da-Costa G, Cotta RMM, Reis JR, SiqueiraBatista R, Gomes AP, Franceschini SCC. Avaliação do cuidado à saúde da gestante no contexto do Programa Saúde da Família. Ciênc Saúde Coletiva 2009; 14 Suppl 1:1347-57.

18. Ministério da Saúde. Portaria no ${ }^{\circ}$. 1886, de 18 de dezembro de 1997. Programas de Agentes Comunitários e Saúde da Família: PACS/PSF. Diário Oficial da União 1997; 22 dez.

19. Ministério da Saúde. Guia prático do Programa Saúde da Família. Brasília: Ministério da Saúde; 2001.

20. Fernandes LC, Bertoldi AD, Barros AJ. Utilização dos serviços de saúde pela população coberta pela Estratégia de Saúde da Família. Rev Saúde Pública 2009; 43:595-603.

21. Goldbaum M, Gianini RG, Novaes HMD, Cesar CLG. Utilização de serviços de saúde em áreas cobertas pelo programa saúde da família (Qualis) no Município de São Paulo. Rev Saúde Pública 2005; 39:1-9.

22. Rodrigues MAP, Facchini LA, Piccini RX, Tomasi E, Thumé E, Silveira DS, et al. Uso de serviços ambulatoriais por idosos nas regiões Sul e Nordeste do Brasil. Cad Saúde Pública 2008; 24:2267-78.
23. Caldeira AP, Oliveira RM, Rodrigues OA. Qualidade da assistência materno-infantil em diferentes modelos de Atenção Primária. Ciênc Saúde Coletiva 2010; 15 Suppl 2:3139-47.

24. Piccini RX, Facchini LA, Tomasi E, Thumé E, Silveira DS, Siqueira FV, et al. Efetividade da atenção pré-natal e de puericultura em unidades básicas de saúde do Sul e do Nordeste do Brasil. Rev Bras Saúde Matern Infant 2007; 7:75-82.

25. Almeida SD, Barros MB. Equidade e atenção à saúde da gestante em Campinas-SP, Brasil. Rev Panam Salud Pública 2005; 17:15-25.

26. Gonçalves CV, Cesar JA, Mendoza-Sassi RA. Qualidade e eqüidade na assistência à gestante: um estudo de base populacional no sul do Brasil. Cad Saúde Pública 2009; 25:2507-16.

27. Cruz SH, Germano JA, Tomasi E, Facchini LA, Piccini RX, Thumé E. Orientações sobre amamentação: a vantagem do Programa de Saúde da Família em municípios gaúchos com mais de 100.000 habitantes no âmbito do Proesf. Rev Bras Epidemiol 2010; 13:259-67.

28. Puccini RF, Pedroso GC, Silva EM, Araujo NS, Silva NN. Eqüidade na atenção pré-natal e ao parto em área da Região Metropolitana de São Paulo, 1996. Cad Saúde Pública 2003; 19:35-45.

29. Almeida CA, Tanaka OY. Perspectiva das mulheres na avaliação do Programa de Humanização do Pré-Natal e Nascimento. Rev Saúde Pública 2009; 43:98-104

30. Bassani DG, Surkan PJ, Olinto MT. Inadequate use of prenatal services among Brazilian women: the role of maternal characteristics. Int Perspect Sex Reprod Health 2009; 35:15-20.

31. Silveira DS, Santos IS. Adequação do pré-natal e peso ao nascer: uma revisão sistemática. Cad Saúde Pública 2004; 20:1160-8.

32. Behague DP, Victora CG, Barros FC. Consumer demand for caesarean sections in Brazil: informed decision making, patient choice, or social inequality? A population based birth cohort study linking ethnographic and epidemiological methods. BMJ 2002; 324:942-5.

33. Dias MAB, Deslandes SF. Cesarianas: percepção de risco e sua indicação pelo obstetra em uma maternidade pública no Município do Rio de Janeiro. Cad Saúde Pública 2004; 20:109-16.

Recebido em 12/Mai/2010

Versão final reapresentada em 02/Fev/2011 Aprovado em 28/Fev/2011 